\title{
Correction: Hou, B. et al. Conceptual Framework and Computational Research of Hierarchical Residential Household Water Demand. Water 2018, 10, 696
}

\author{
Wenxiang Pan ${ }^{1,+}$, Baodeng Hou ${ }^{2,+}\left(\mathbb{D}\right.$, Ruixiang Yang ${ }^{3}$, Xuzhu Zhan ${ }^{4}$, Wenkai Tian ${ }^{5}$, Baoqi Li ${ }^{2}$, \\ Weihua Xiao $^{2}$, Jianhua Wang ${ }^{2, *}$, Yuyan Zhou ${ }^{2}$ (D), Yong Zhao ${ }^{2}$ (D) and Xuerui Gao ${ }^{1, *(D)}$ \\ 1 Institute of Soil and Water Conservation of Northwest A\&F University, Yangling 712100, China; \\ panwx1992@126.com \\ 2 State Key Laboratory of Simulation and Regulation of Water Cycle in River Basin, \\ China Institute of Water Resources and Hydropower Research, Beijing 100038, China; \\ houbaodeng@163.com (B.H.); tongyeti@163.com (B.L.); xwsen998@163.com (W.X.); \\ zhouyuyan1@126.com (Y.Z.); zhaoyong@iwhr.com (Y.Z.) \\ 3 College of Water Resource and Hydropower, Sichuan University, Chengdu 610065, China; \\ smsxfypy@163.com \\ 4 Beijing Water Consulting Co., Ltd., Beijing 100044, China; zhanxuzhu@163.com \\ 5 North China University of Water Resources and Electric Power, Zhengzhou 450045, China; \\ 17600131539@163.com \\ * Correspondence: jhwangiwhr@126.com (J.W.); gaoxuerui666@163.com (X.G.) \\ + These authors contributed equally to this work.
}

Received: 30 July 2018; Accepted: 21 August 2018; Published: 1 September 2018

check for updates

We wish to make the following correction to the published paper [1]. Two authors have been added and the author contributions and acknowledgments have been updated. The correct versions are:

Wenxiang Pan ${ }^{1,+}$, Baodeng Hou ${ }^{2,+}$, Ruixiang Yang ${ }^{3}$, Xuzhu Zhan ${ }^{4}$, Wenkai Tian ${ }^{5}$, Baoqi Li ${ }^{2}$, Weihua Xiao ${ }^{2}$, Jianhua Wang ${ }^{2, *}$, Yuyan Zhou ${ }^{2}$, Yong Zhao ${ }^{2}$ and Xuerui Gao ${ }^{1, *}$

1 Institute of Soil and Water Conservation of Northwest A\&F University, Yangling 712100, China; panwx1992@126.com

2 State Key Laboratory of Simulation and Regulation of Water Cycle in River Basin, China Institute of Water Resources and Hydropower Research, Beijing 100038, China; houbaodeng@163.com (B.H.); tongyeti@163.com (B.L.); xwsen998@163.com (W.X.); zhouyuyan1@126.com (Y.Z.); zhaoyong@iwhr.com (Y.Z.)

3 College of Water Resource and Hydropower, Sichuan University, Chengdu 610065, China; smsxfypy@163.com

$4 \quad$ Beijing Water Consulting Co., Ltd., Beijing 100044, China; zhanxuzhu@163.com

5 North China University of Water Resources and Electric Power, Zhengzhou 450045, China; 17600131539@163.com

* Correspondence: jhwangiwhr@126.com (J.W.); gaoxuerui666@163.com (X.G.)

$+\quad$ These authors contributed equally to this work.

Author Contributions: Wenxiang Pan, Baodeng Hou and Ruixiang Yang conceived and designed the methods and model; Jianhua Wang and Yong Zhao conceived the theory; Wenxiang Pan, Xuzhu Zhan and Wenkai Tian carried out the experimental observation; Wenxiang Pan, Baoqi Li and Yuyan Zhou analyzed the data; Xuerui Gao, Weihua Xiao and Jianhua Wang contributed analysis tools; Baodeng Hou and Ruixiang Yang wrote the paper. Wenxiang Pan and Baodeng Hou contributed equally to this work and should be considered co-first authors.

Acknowledgments: This research was financially supported by the National Natural Science Foundation of China $(51509267,51625904,51779271$ and 51409275) and the IWHR Research \& Development Support Program (WR0145B622017). 
The reasons for these changes are as follows. When Xuerui Gao worked in China Institute of Water Resources and Hydropower Research, he worked in the same research group as the other authors. When he was transferred to Northwest A\&F University, he and his master's student Wenxiang Pan continued to engage in and contribute to the research. Therefore, it was an oversight not to have included them on the original paper and we are pleased to recognize their input and add their names to the author list. We would like to apologize for any inconvenience caused by these changes.

The manuscript will be updated and the original will remain online on the article webpage, with a reference to this Correction.

\section{Reference}

1. Hou, B.; Yang, R.; Zhan, X.; Tian, W.; Li, B.; Xiao, W.; Wang, J.; Zhou, Y.; Zhao, Y. Conceptual Framework and Computational Research of Hierarchical Residential Household Water Demand. Water 2018, 10, 696. [CrossRef]

(C) 2018 by the authors. Licensee MDPI, Basel, Switzerland. This article is an open access article distributed under the terms and conditions of the Creative Commons Attribution (CC BY) license (http:/ / creativecommons.org/licenses/by/4.0/). 